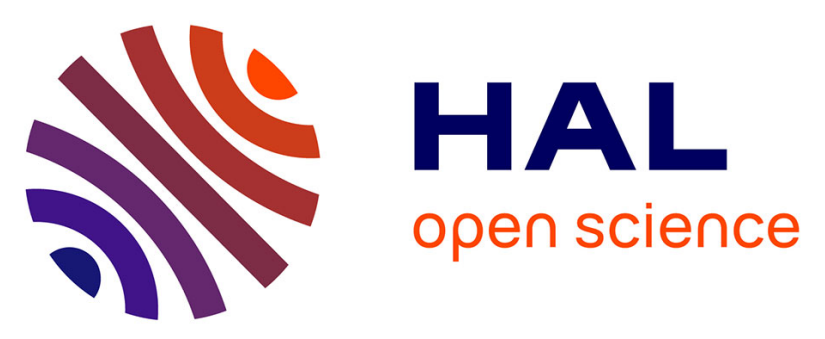

\title{
Banking Crises in Developing Countries-What Crucial Role of Exchange Rate Stability and External Liabilities?
}

Brahim Gaies, Stéphane Goutte, Khaled Guesmi

\section{- To cite this version:}

Brahim Gaies, Stéphane Goutte, Khaled Guesmi. Banking Crises in Developing Countries-What Crucial Role of Exchange Rate Stability and External Liabilities?. 2019. hal-01968084

\section{HAL Id: hal-01968084 \\ https://hal.science/hal-01968084}

Preprint submitted on 9 Jan 2019

HAL is a multi-disciplinary open access archive for the deposit and dissemination of scientific research documents, whether they are published or not. The documents may come from teaching and research institutions in France or abroad, or from public or private research centers.
L'archive ouverte pluridisciplinaire HAL, est destinée au dépôt et à la diffusion de documents scientifiques de niveau recherche, publiés ou non, émanant des établissements d'enseignement et de recherche français ou étrangers, des laboratoires publics ou privés. 


\title{
Banking Crises in Developing Countries-What Crucial Role of Exchange Rate Stability and External Liabilities?
}

\author{
Brahim GAIES \\ IPAG Lab - IPAG Business School, Paris, France \\ Stéphane GOUTTE \\ University Paris 8, LED, France and Paris Business School, PSB, Paris, France \\ Khaled GUESMI \\ IPAG Lab, IPAG Business School, Paris, France and Telfer School of Management, \\ University of Ottawa, Canada
}

\begin{abstract}
We examine the determinants of banking crises occurrence in developing countries, focusing on the impact of the nature of external liabilities and exchange rate stability. For this purpose, we use a logit panel model, including 67 developing countries observed between 1972 and 2011, as well as a set of alternative estimation methods (logit fixed-effects and probit randomeffects) and robustness tests. We find that FDI liabilities reduce the occurrence of banking crises, but debt liabilities increase them. In addition, banking crises occurrence decreases in developing countries with the stability of the exchange rate, real GDP growth, as well as better human capital quality and better political institutions.
\end{abstract}

Keywords: Financial Crises, External Liabilities, Exchange Rate Stability JEL: G01, G15, F31, O24

\section{Introduction}

Although the literature on the determinants of banking crises in developed and emerging countries is abundant, academic work for the case of developing countries is relatively rare (Babecký et al., 2014; Bordo, 2018; Gaies et al., 2018). It seems that crises in non-emerging developing countries may be overshadowed by the global financial crisis 2007-2008 and the crises in emerging countries (Joyce, 2010). Yet, since the mid-1980s, the number of banking crises in developing countries has become significant (see, Figure A in appendix), especially after their accession to the phenomenon of financial globalization and their exchange rate regime reforms (Wei, 2018). This factual reality highlights the relevance of two questions: i) Should developing countries be more integrated into financial globalization, regarding the relationship between external liabilities and banking crises? ii) and as a corollary, what financial liberalization and exchange rate policies should they choose in the context of financial globalization?

Using a logit panel model, including 67 developing countries observed between 1972 and 2011, as well as a set of alternative estimation methods (logit fixed-effects and probit randomeffects) and robustness tests, this article aims to examine these two questions. By doing so, it enriches the literature on the determinants of crises in non-emerging developing, while focusing on the role of exchange rate stability and external liabilities. The main results of the study indicate that FDI liabilities decrease banking crises occurrence. Conversely, external debt liabilities increase it. Also, the occurrence of banking crises decreases with exchange rate stability, real GDP growth, as well as better human capital quality and better political institutions. These results lead us to conclude that less volatile exchange rate regimes and 
capital controls are the right political choice for developing countries to combat financial instability in the context of financial globalization.

\section{Recent literature and main contributions}

As regards recent literature on the relationship between financial openness and banking crises, we note that Joyce (2011) examined 20 emerging countries in the period 1976-2002. He shows that external debts promote the incidence of banking crises, whereas FDI and portfolio investments reduce their occurrence. In the same vein, the study of Ahrend and Goujard (2014) emphasizes the positive effect of foreign debts in emerging economies on banking crises incidence. Caballero (2015) proves that the level of financial integration of domestic banks is a determinant of the occurrence of banking crises. Though, Gourinchas and Obstfeld (2012) nuance these results, by showing that there is no significant relationship between the share of external debt in total external liabilities and the probability of banking crises in emerging markets. Later, in an empirical study, Lane and McQuade (2014) find that there is a strong correlation between net inflows of external debt and the domestic credit boom, observed for 54 developed and emerging countries from 1993 to 2008. On the contrary, FDI flows are uncorrelated with this boom. Also, Hamdi and Boukef Jlassi (2014) studied 58 developing countries during the period 1984-2007.Their conclusion is that foreign debt liabilities to total liabilities and foreign direct investment liabilities to total liabilities increase banking crises incidence. Two years later, Lee et al. (2016) examine the case of 39 emerging, developing and developed countries. They conclude that all foreign private flows have a negative impact on the occurrence of banking crises. Conversely, Caballero (2016) finds a positive relationship between capital inflow and banking crises occurrence for 141 countries between 1973 and 2008.

In comparison with these recent studies, our article goes a step further in three main respects.

- First, by using the exchange rate stability index and the "Z-score" of exchange rate volatility, our study completes academic work that explains the relationships between external financing and banking crises, but which did not include exchange rate effects.

- Second, compared with recent work, in this article, we observe the longest period ever studied (including the period after the global financial crisis of 2007-2008), and we focused on the case of non-emerging developing countries, knowing that current literature on crises has traditionally been concentrating on emerging countries or heterogeneous samples of countries, including developing and developed economies, as already noted in the review of recent literature by Babecký et al. (2014).

- Third, the empirical analysis of the article is based on three estimators (logit randomeffects, logit fixed-effects, and probit random-effects) and a battery of sensibility tests, while the most recent studies use only one estimator for their estimates. 


\section{Method}

\subsection{Sample selection}

We utilize unbalanced panel data for 67 economies among low- and middle-income countries $^{1}$, by referring to the World Bank classification, between 1972 and $2011^{2}$, in yearly observations $^{3}$, to provide a sample representation of non-emerging developing countries ${ }^{4}$. By doing that, we include most non-emerging developing countries and observe the longest period studied, compared with recent work cited above.

\subsection{Variables}

To measure the occurrence of crises, we consider the binary variable of banking crises ${ }^{5}$ (BCRISES) developed by Laeven and Valencia (2008) as our dependent variable, following Hamdi and Boukef Jlassi (2014), Caballero (2015), Caballero (2016) and Lee et al. (2016). BCRISES takes (1) if country $i$ in period $t$ experiences a crisis and (0) otherwise.

Furthermore, BCRISES is explained by several measures of external liabilities and exchange rate stability, selected from the External Wealth of Nations Dataset (EWN), that constitute the set of interest variables, as well as five control variables extracted from the World Development Indicators database (WDI). All these independent variables are one period lagged (L.) because we target to identify the early warning indicators of banking crises in the long term, as done in the model of Lee et al. (2016). In addition to that, this choice obviates the endogeneity problems, according to Caballero (2016).

The independent variables (interest and control variables) are presented in Table 1 below.

\footnotetext{
${ }^{1}$ Sample countries in appendix.

${ }^{2}$ We fixed our spaciotemporal framework under the constraint of the availability of data, especially for External Wealth of Nations Dataset (EWN, last extended in 2014), which is characterized by too much missing data (blanks) for the years 2012, 2013 and 2014, knowing that the interest variables used in our models are extracted from this database. Consequently, we stopped our analysis in 2011 to prevent a problem of biased estimations due to too strongly unbalanced data across countries.

${ }^{3}$ Summary statistics in appendix.

${ }^{4}$ The World Bank considers that a country is low-income if its GNI per capita is lower or equal to 935 US dollars. A country is considered to have lower average income if its GNI per capita is no less than 936 US dollars and no more than 3705 US dollars, a country has a higher average income if its GNI per capita is between 3706 US dollars and 11455 US dollars, and a country is at higher income if its GNI per capita exceeds 11456 US dollars. The countries with higher average income and higher income are characterized by a level of economic and financial development and institutional quality that is higher than that of other countries. These two groups mainly comprise developed and emerging economies. Thus, by excluding them from our sample, we enhance the homogeneity of the latter. Also, we have removed five middle-income countries from our sample: Egypt, Pakistan, India, Indonesia, and the Philippines because they are classified as emerging economies by Morgan Stanley Capital International (MSCI) 2018 indices. Furthermore, the number of country groups studied is often lower than 67 countries and varies from one regression to another because we use an unbalanced panel.

${ }^{5}$ Systemic Banking Crises Database, IMF (2012).
} 
Table 1 Independent variables

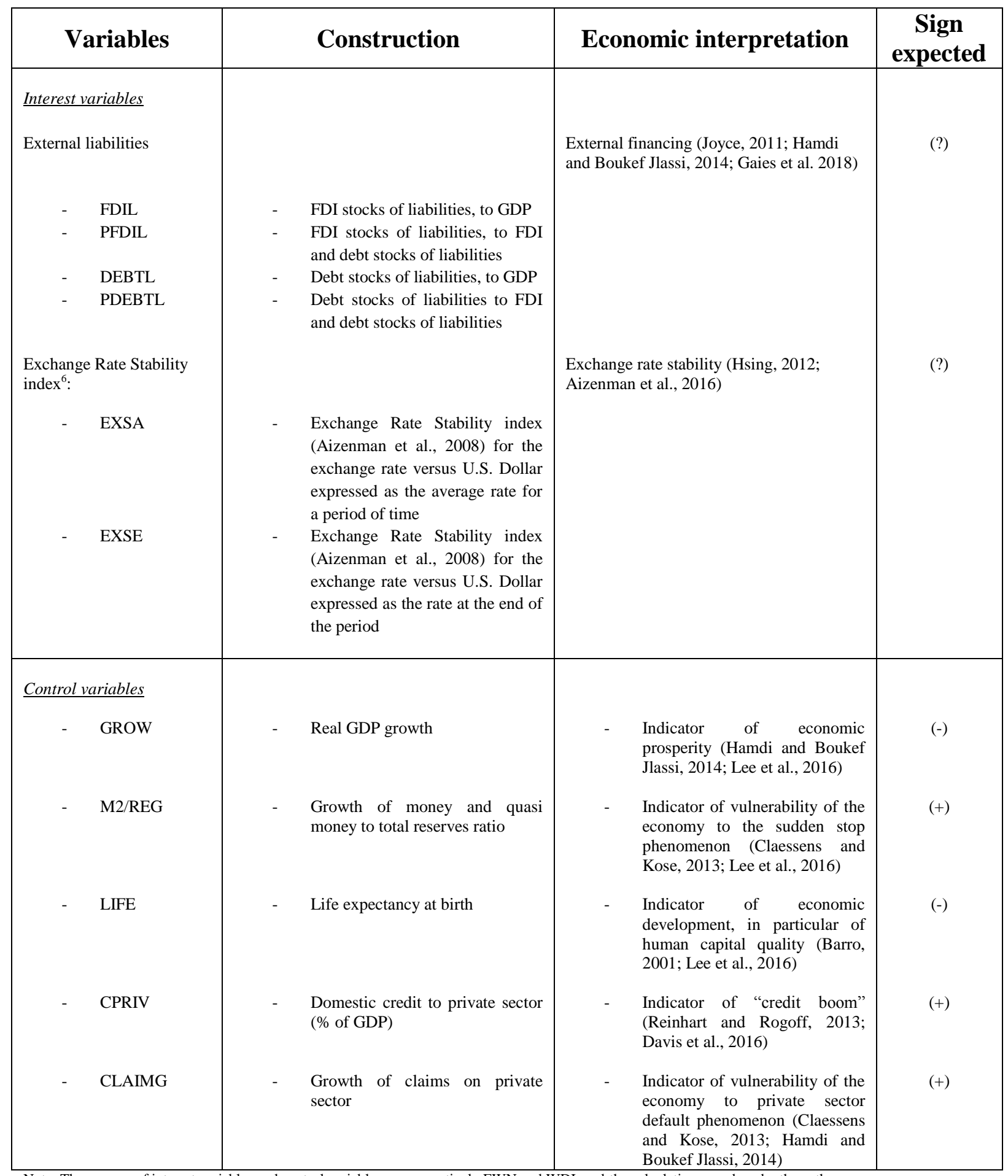

Note: The sources of interest variables and control variables are respectively EWN and WDI, and the calculations are done by the authors.

${ }^{6} E X S A$ or $E X S E=\frac{0.01}{0.01+\operatorname{stdev}(\Delta(\log (\text { exch_rate }))}$, when, instability $=0 \leq$ value of $($ EXSA or EXSE $) \leq 1=$ stability. 


\subsection{Estimation methods}

We refer to Joyce (2011), Hamdi and Boukef Jlassi (2014) and Caballero (2016) in employing a random-effects logit model $^{7}$ for our baseline estimations. We also use a fixed-effects logit model $^{8}$ and a random-effects probit ${ }^{9}$ model to test the robustness of our baseline estimations. Knowing that the dependent variable BCRISES is binary, our model is expressed as drawn below in Eq. (1):

$$
\text { Zit }=\varphi K_{i t}+\lambda_{i}+v_{i t}
$$

Where $Z$ indicates whether country $i$ experienced a banking crisis during the year $t$. $K$ represents the explanatory variables presented above and $\lambda$ indicates the country effects. $v$ is the independent error term identically distributed with mean 0 and variance $\sigma^{2}$. $i=1 \ldots N$ countries and $t=1 \ldots T$ years. If the economy of country $i$ endured a banking crisis in the year $t, Z_{i t}$ is positive. Hence,

BCRISIS $=\left\{\begin{array}{l}1, Z_{i t}>0 \\ 0, \text { otherwise. }\end{array}\right.$

\section{Results and robustness}

\subsection{Results}

Table 2 below shows the results of our baseline estimations. It highlights that the magnitude of the effect of external liabilities based on debt (DEBTL and PDEBTL) on the occurrence of banking crises is relatively large: the debt liabilities are a significant positive determinant of banking crises in developing countries. Similarly, the coefficients of external liabilities indicators through FDI (FDIL and PFDIL) indicate that the magnitude of the effect of FDI liabilities on the occurrence of banking crises is also relatively large, which reveals that this type of external financing reduces significantly the occurrence of banking crises in developing countries.

In sum, these results are corroborated by the studies of Joyce (2011), Hamdi and Boukef Jlassi (2014) and Caballero (2016) for spatiotemporal horizons different from ours. However, they nuance the findings of Lee et al. (2016), who do not differentiate external financing according to its nature.

Moreover, these results can be explained by the fact that the presence of foreign investors allows the diversification and reduction of financial and monetary risks (see e.g. Bekaert and Harvey, 2000). On the contrary, external indebtedness is often identified as a source of agency problems, macro-instabilities and debt default (see e.g. McKinnon and Pill, 1996, 1998; Broner et al., 2010).

In addition, in the outputs of Table 2, it appears that the exchange rate stability indicators (EXSA and EXSE) have a statistically significant negative effect on the occurrence of

\footnotetext{
${ }^{7}$ (Stata xtlogit, re command).

8 (Stata xtlogit, fe command).

${ }^{9}$ (Stata xprobit, re command).
} 
banking crises. The magnitude of this effect is very high compared to other dependent variables, which

means that the exchange rate stability reduces considerably the occurrence of banking crises in developing countries. This result can be explained by the fact that a large exchange rate fluctuation may be synonymous with increased uncertainty (see e.g. Rose, 2000; Frankel and Rose, 2002).

Finally, Table 2 shows that the economic prosperity measured by GROW and the human capital quality indicated by LIFE are the robust decreasing factors of banking crises. Conversely, the private credits measured by CPRIV are likely to increase the occurrence of banking crises.

\subsection{Robustness}

To further test the sensibility of our baseline estimations, we established several robustness tests as follows:

i) We re-estimated our crises model presented in Eq. (1) using a random-effects probit model and a fixed-effects logit model. Tables 3 and 4 below regroup the results of this test. The latter confirms our basic conclusions obtained from the outputs of Table 2 .

ii) We included two alternative variables of exchange rate stability based on the measure of the de facto exchange rate volatility "Z-score" proposed by Ghosh et al. (2003). They are as follows:

$Z-S C O E X A$ or $Z-S C O E X E=\sqrt{\mu_{t}^{2}+\sigma_{t}^{2}}$

Where $\mu$ and $\sigma$ are respectively the two-year average and standard deviation of the exchange rate versus U.S. Dollar expressed as the average rate for a period of time (Z-SCOEXA) and as the rate at the end of the period (Z-SCOEXE). To our knowledge, no study has yet included this indicator to examine the determinants of banking crises in developing countries. Table 5 below indicates that the sign, significance and magnitude of the coefficients of these exchange rate volatility variables, and those of the other independent variables confirm also our main findings.

iii) When we considered a shorter sample period, excluding respectively sub-periods characterized by the highest banking crises occurrence (see, Figure A in appendix), namely 1997-2001 and 1982-1986, the main results basically hold, as Tables A and $\mathrm{B}$ in appendix highlight. We obtained a similar result when we remove the sub-period 2007-2011, which corresponds to the world financial crisis period, because this crisis influenced more developed and emerging economies than developing economies, according to Reinhart and Rogoff (2013).The same conclusion is drawn, as per Tables $\mathrm{D}$ and $\mathrm{E}$ in appendix, when we considered a smaller sample of countries, omitting two sub-groups also characterized by the highest occurrence of banking crises, namely "South Asia, East Asia and Pacific" at first, then "Latin America, Caribbean, Europe and Central Asia". 
Table 2 Baseline estimations: Logit random-effects

Table 4 Robustness estimations: Logit fixed-effects

\begin{tabular}{|c|c|c|c|c|c|c|c|c|}
\hline \multirow{3}{*}{$\begin{array}{l}\text { LIA1/ LIA2: } \\
\text { L.GROW }\end{array}$} & \multicolumn{2}{|c|}{ FDIL } & \multicolumn{2}{|c|}{ PFDIL } & \multicolumn{2}{|c|}{ DEBTL } & \multicolumn{2}{|c|}{ PDETL } \\
\hline & (1) & (2) & (3) & (4) & (5) & (6) & (7) & (8) \\
\hline & $\begin{array}{c}-0.058 * * * \\
(0.021)\end{array}$ & $\begin{array}{c}-0.055^{* * * *} \\
(0.021)\end{array}$ & $\begin{array}{c}-0.048 * * \\
(0.022)\end{array}$ & $\begin{array}{c}-0.046^{* * *} \\
(0.022)\end{array}$ & $\begin{array}{c}-0.050 * * \\
(0.021)\end{array}$ & $\begin{array}{c}-0.049 * * \\
(0.021)\end{array}$ & $\begin{array}{c}-0.051 * * \\
(0.021)\end{array}$ & $\begin{array}{c}-0.049 * * \\
(0.022)\end{array}$ \\
\hline \multirow[t]{2}{*}{ L.M2/REG } & -0.003 & -0.001 & 0.074 & 0.068 & 0.067 & 0.061 & 0.017 & 0.018 \\
\hline & $(0.210)$ & $(0.210)$ & $(0.211)$ & $(0.211)$ & $(0.211)$ & $(0.211)$ & $(0.210)$ & $(0.210)$ \\
\hline \multirow[t]{2}{*}{ L.CLAIMG } & 0.001 & 0.001 & 0.001 & 0.001 & 0.001 & 0.001 & 0.001 & 0.001 \\
\hline & $(0.001)$ & $(0.001)$ & $(0.001)$ & $(0.001)$ & $(0.001)$ & $(0.001)$ & $(0.001)$ & $(0.001)$ \\
\hline \multirow[t]{2}{*}{ L.LIFE } & $-0.056^{* * * *}$ & $-0.056^{* * *}$ & $-0.060 * *$ & $-0.061 * *$ & $-0.064 * * *$ & $-0.063^{* * *}$ & $-0.058 * * *$ & $-0.058 * * *$ \\
\hline & $(0.021)$ & $(0.021)$ & $(0.024)$ & $(0.024)$ & $(0.022)$ & $(0.021)$ & $(0.021)$ & $(0.021)$ \\
\hline \multirow[t]{2}{*}{ L.CPRIV } & $0.389 *$ & $0.393^{*}$ & $0.559^{* *}$ & $0.545^{* *}$ & $0.423 * *$ & $0.420^{* *}$ & $0.409^{* *}$ & $0.412 * *$ \\
\hline & $(0.208)$ & $(0.206)$ & $(0.230)$ & $(0.228)$ & $(0.209)$ & $(0.209)$ & $(0.206)$ & $(0.206)$ \\
\hline L.LIA1 & $\begin{array}{c}-\mathbf{- 0 . 2 9 7} * * \\
(0.143)\end{array}$ & $\begin{array}{c}-\mathbf{- 0 . 2 9 8} * * \\
(0.142)\end{array}$ & & & $\begin{array}{c}\mathbf{0 . 5 7 4} * * * \\
(0.202)\end{array}$ & $\begin{array}{c}\mathbf{0 . 5 2 6} * * * * \\
(0.203)\end{array}$ & & \\
\hline \multirow[t]{2}{*}{ L.EXSE } & $-2.320 * * *$ & & $-1.955 * *$ & & $-1.769 * *$ & & $-2.020 * *$ & \\
\hline & $(0.815)$ & & $(0.800)$ & & $(0.817)$ & & $(0.791)$ & \\
\hline \multirow[t]{2}{*}{ L. EXSA } & & $-2.827 * * *$ & & $-2.259 * * *$ & & $-2.123 * *$ & & $-2.443 * * *$ \\
\hline & & $(0.879)$ & & $(0.844)$ & & $(0.868)$ & & $(0.847)$ \\
\hline L. LIA2 & & & $\begin{array}{c}\mathbf{- 0 . 7 6 4 * * *} \\
(0.204)\end{array}$ & $\begin{array}{c}\mathbf{- 0 . 7 1 2} * * * * \\
(0.198)\end{array}$ & & & $\begin{array}{c}1.594 * * * \\
(0.600)\end{array}$ & $\begin{array}{l}\mathbf{1 . 5 1 9} * * \\
(0.594)\end{array}$ \\
\hline \multirow[t]{2}{*}{ Constant } & 0.122 & 0.212 & 0.939 & 0.946 & $-2.738^{*}$ & $-2.504 *$ & $-7.534 * *$ & $-7.126 * *$ \\
\hline & $(1.182)$ & (1.176) & $(1.370)$ & $(1.343)$ & $(1.492)$ & (1.487) & (3.005) & (2.967) \\
\hline Observations & 1,569 & 1,569 & 1,569 & 1,569 & 1,586 & 1,586 & 1,586 & 1,586 \\
\hline $\begin{array}{l}\text { Wald Test } \\
\text { Statistic }\end{array}$ & 34.07 & 35.97 & 39.72 & 41.41 & 37.65 & 38.79 & 36.53 & 38.17 \\
\hline Log-Likelihood & -318 & -316.3 & -311.1 & -310.3 & -315.9 & -315 & -316.1 & -314.7 \\
\hline $\begin{array}{l}\text { Likelihood } \\
\text { Ratio Test }\end{array}$ & 21.66 & 21.56 & 31.52 & 30.16 & 23.88 & 23.16 & 23.56 & 22.86 \\
\hline
\end{tabular}

Notes: Dependent variable is a banking crisis dummy. Marginal effects are reported. Symbols $* * *$ and $* * *$ indicate statistical significance at $10 \%, 5 \%$, and $1 \%$, respectively. Standard errors are presented below the corresponding

Table 3 Robustness estimations: Probit random-effects

Table 5 Robustness estimations:-Alternative variables of exchange

\begin{tabular}{|c|c|c|c|c|c|c|c|c|}
\hline \multirow{3}{*}{$\begin{array}{l}\text { LIA1/ LIA2: } \\
\text { L.GROW }\end{array}$} & \multirow{2}{*}{\multicolumn{2}{|c|}{$\begin{array}{l}\text { Lande } \\
\text { Fate stabifity }\end{array}$}} & \multicolumn{2}{|c|}{ PFDIL } & \multicolumn{4}{|c|}{$\begin{array}{l}\text { Allernative varlables of excnange } \\
\text { DEBTL }\end{array}$} \\
\hline & & & (3) & (4) & (5) & (6) & (7) & (8) \\
\hline & $\begin{array}{c}-0.033^{* * * *} \\
(0.011)\end{array}$ & $\begin{array}{c}-0.032^{* * * *} \\
(0.011)\end{array}$ & $\begin{array}{c}-0.029 * * \\
(0.012)\end{array}$ & $\begin{array}{c}-0.028^{* *} \\
(0.012)\end{array}$ & $\begin{array}{c}-0.030^{* * * *} \\
(0.011)\end{array}$ & $\begin{array}{c}-0.029 * * * \\
(0.011)\end{array}$ & $\begin{array}{c}-0.029^{* * *} * \\
(0.011)\end{array}$ & $\begin{array}{c}-0.028^{* *} \\
(0.011)\end{array}$ \\
\hline \multirow[t]{2}{*}{ L.M2/REG } & -0.017 & -0.017 & 0.022 & 0.020 & 0.019 & 0.017 & -0.006 & -0.006 \\
\hline & $(0.111)$ & $(0.111)$ & $(0.113)$ & $(0.113)$ & $(0.112)$ & $(0.112)$ & $(0.112)$ & $(0.112)$ \\
\hline \multirow[t]{2}{*}{ L.CLAIMG } & 0.000 & 0.000 & 0.000 & 0.000 & 0.000 & 0.000 & 0.000 & 0.000 \\
\hline & $(0.000)$ & $(0.000)$ & $(0.000)$ & $(0.000)$ & $(0.000)$ & $(0.000)$ & $(0.000)$ & $(0.000)$ \\
\hline \multirow[t]{2}{*}{ L.LIFE } & $-0.029 * * *$ & $-0.029 * * *$ & $-0.030^{* *}$ & $-0.030 * *$ & $-0.032 * * *$ & $-0.032 * * *$ & $-0.029 * * *$ & $-0.029 * * *$ \\
\hline & $(0.011)$ & $(0.011)$ & $(0.012)$ & $(0.012)$ & $(0.011)$ & $(0.011)$ & $(0.011)$ & $(0.011)$ \\
\hline \multirow[t]{2}{*}{ L.CPRIV } & $0.181^{*}$ & $0.182^{*}$ & $0.264 * *$ & $0.256^{* *}$ & $0.176^{*}$ & $0.174 *$ & $0.197 *$ & $0.196^{*}$ \\
\hline & $(0.102)$ & $(0.102)$ & $(0.114)$ & $(0.113)$ & $(0.100)$ & $(0.100)$ & $(0.101)$ & $(0.101)$ \\
\hline L.LIA1 & $\begin{array}{c}-\mathbf{- 0 . 1 5 6 * *} \\
(0.074)\end{array}$ & $\begin{array}{c}\mathbf{- 0 . 1 5 6 * *}^{* *} \\
(0.073)\end{array}$ & & & $\begin{array}{c}\mathbf{0 . 2 7 3}^{* * * *} \\
(0.097)\end{array}$ & $\begin{array}{c}\mathbf{0 . 2 5 7} * * * \\
(0.098)\end{array}$ & & \\
\hline \multirow[t]{2}{*}{ L.EXSE } & $-0.951 * * *$ & & $-0.803 * *$ & & $-0.699 * *$ & & $-0.831 * *$ & \\
\hline & $(0.329)$ & & $(0.336)$ & & $(0.335)$ & & $(0.325)$ & \\
\hline \multirow[t]{2}{*}{ L. EXSA } & & $-1.130 * * *$ & & $-0.916 * * *$ & & $-0.837 * *$ & & $-0.992 * * *$ \\
\hline & & $(0.351)$ & & $(0.353)$ & & $(0.354)$ & & $(0.345)$ \\
\hline L. LIA2 & & & $\begin{array}{c}-\mathbf{0 . 3 8 7 * * * *} \\
(0.105)\end{array}$ & $\begin{array}{c}\mathbf{- 0 . 3 6 6 * * * *} \\
(0.103)\end{array}$ & & & $\begin{array}{c}\mathbf{0 . 7 9 8} * * * * \\
(0.287)\end{array}$ & $\begin{array}{c}\mathbf{0 . 7 7 9} * * * * \\
(0.286)\end{array}$ \\
\hline \multirow[t]{2}{*}{ Constant } & -0.032 & -0.004 & 0.360 & 0.363 & $-1.355^{*}$ & $-1.281 *$ & $-3.910^{* * *}$ & $-3.798 * * *$ \\
\hline & $(0.584)$ & $(0.583)$ & $(0.679)$ & $(0.669)$ & $(0.716)$ & $(0.716)$ & $(1.472)$ & $(1.461)$ \\
\hline Observations & 1,569 & 1,569 & 1,569 & 1,569 & 1,586 & 1,586 & 1,586 & 1,586 \\
\hline $\begin{array}{l}\text { Wald Test } \\
\text { Statistic }\end{array}$ & 34.16 & 35.77 & 38.71 & 40.25 & 37.98 & 39 & 37.37 & 38.81 \\
\hline Log-Likelihood & -317 & -315.7 & -310.3 & -309.8 & -315.3 & -314.5 & -314.9 & -313.9 \\
\hline $\begin{array}{l}\text { Likelihood Ratio } \\
\text { Test }\end{array}$ & 24.87 & 24.77 & 34.41 & 33.37 & 26.19 & 25.74 & 27.16 & 26.67 \\
\hline
\end{tabular}

coefficient. 


\begin{tabular}{|c|c|c|c|c|c|c|c|c|}
\hline \multirow{3}{*}{$\begin{array}{l}\text { LIA1/ LIA2: } \\
\text { L.GROW }\end{array}$} & \multicolumn{2}{|c|}{ FDIL } & \multicolumn{2}{|c|}{ PFDIL } & \multicolumn{2}{|c|}{ DEBTL } & \multicolumn{2}{|c|}{ PDETL } \\
\hline & (1) & (2) & (3) & (4) & (5) & (6) & (7) & (8) \\
\hline & $\begin{array}{c}-0.057 * * * \\
(0.022)\end{array}$ & $\begin{array}{c}-0.053^{* *} \\
(0.022)\end{array}$ & $\begin{array}{l}-0.040^{*} \\
(0.022)\end{array}$ & $\begin{array}{l}-0.039^{*} \\
(0.022)\end{array}$ & $\begin{array}{c}-0.048^{* *} \\
(0.023)\end{array}$ & $\begin{array}{c}-0.047 * * \\
(0.023)\end{array}$ & $\begin{array}{c}-0.047^{* * *} \\
(0.022)\end{array}$ & $\begin{array}{c}-0.045^{* *} \\
(0.022)\end{array}$ \\
\hline L.M2/REG & -0.000 & -0.006 & 0.111 & 0.098 & 0.077 & 0.069 & 0.029 & 0.023 \\
\hline & $(0.207)$ & $(0.206)$ & $(0.211)$ & $(0.210)$ & $(0.209)$ & $(0.208)$ & $(0.208)$ & $(0.207)$ \\
\hline L.CLAIMG & 0.001 & 0.001 & 0.001 & 0.001 & 0.001 & 0.001 & 0.001 & 0.001 \\
\hline L.LIFE & $\begin{array}{l}(0.000) \\
-0.009 \\
(0.035)\end{array}$ & $\begin{array}{l}(0.000) \\
-0.012 \\
(0.036)\end{array}$ & $\begin{array}{c}(0.000) \\
-0.019 \\
(0.039)\end{array}$ & $\begin{array}{l}(0.000) \\
-0.020 \\
(0.039)\end{array}$ & $\begin{array}{c}(0.000) \\
-0.036 \\
(0.038)\end{array}$ & $\begin{array}{l}(0.000) \\
-0.036 \\
(0.038)\end{array}$ & $\begin{array}{c}(0.000) \\
-0.014 \\
(0.036)\end{array}$ & $\begin{array}{c}(0.000) \\
-0.016 \\
(0.037)\end{array}$ \\
\hline L.CPRIV & $\begin{array}{c}0.718^{* * * *} \\
(0.249)\end{array}$ & $\begin{array}{c}0.709^{* * * *} \\
(0.249)\end{array}$ & $\begin{array}{c}0.862 * * * \\
(0.273)\end{array}$ & $\begin{array}{c}0.843^{* * * *} \\
(0.271)\end{array}$ & $\begin{array}{c}0.678 * * * \\
(0.254)\end{array}$ & $\begin{array}{c}0.674 * * * \\
(0.254)\end{array}$ & $\begin{array}{c}0.684 * * * \\
(0.252)\end{array}$ & $\begin{array}{c}0.686^{* * * *} \\
(0.253)\end{array}$ \\
\hline L.LIA1 & $\begin{array}{c}-\mathbf{- 0 . 5 4 3} * * * \\
(0.187)\end{array}$ & $\begin{array}{c}-\mathbf{- 0 . 5 2 8} * * * * \\
(0.185)\end{array}$ & & & $\begin{array}{c}\mathbf{0 . 7 3 9} * * * \\
(0.238)\end{array}$ & $\begin{array}{c}\mathbf{0 . 6 9 5} * * * \\
(0.240)\end{array}$ & & \\
\hline L.EXSE & $\begin{array}{c}\mathbf{- 2 . 1 5 0} * * * \\
(0.836)\end{array}$ & & $\begin{array}{l}-\mathbf{1 . 5 7 3} * \\
(0.814)\end{array}$ & & $\begin{array}{l}-\mathbf{1 . 5 1 8}^{*} \\
(0.878)\end{array}$ & & $\begin{array}{c}\mathbf{- 1 . 7 2 2 * * *} \\
(0.837)\end{array}$ & \\
\hline L. EXSA & & $\begin{array}{c}\mathbf{- 2 . 5 2 7} * * * * \\
(0.865)\end{array}$ & & $\begin{array}{l}-\mathbf{1 . 5 9 7 *} \\
(0.864)\end{array}$ & & $\begin{array}{c}-\mathbf{1 . 7 6 6 * * *} \\
(0.898)\end{array}$ & & $\begin{array}{c}-\mathbf{2 . 0 4 5} * * \\
(0.867)\end{array}$ \\
\hline L. LIA2 & & & $\begin{array}{c}-\mathbf{1 . 3 1 9 * * * *} \\
(0.262)\end{array}$ & $\begin{array}{c}-\mathbf{1 . 2 4 0} * * * * \\
(0.257)\end{array}$ & & & $\begin{array}{c}\mathbf{1 . 9 2 2} * * * * \\
(0.701)\end{array}$ & $\begin{array}{c}\mathbf{1 . 8 2 2} * * * * \\
(0.697)\end{array}$ \\
\hline Observations & 832 & 832 & 832 & 832 & 832 & 832 & 832 & 832 \\
\hline $\begin{array}{l}\text { Wald Test } \\
\text { Statistic }\end{array}$ & 37.57 & 39.66 & 60.82 & 60.23 & 40.16 & 41.08 & 38.57 & 40.04 \\
\hline Log-Likelihood & -219.3 & -218.2 & -207.6 & -207.9 & -218 & -217.5 & -218.8 & -218 \\
\hline
\end{tabular}

\begin{tabular}{|c|c|c|c|c|c|c|c|c|}
\hline \multirow{3}{*}{$\begin{array}{l}\text { LIA1/ LIA2: } \\
\text { L.GROW }\end{array}$} & \multicolumn{2}{|c|}{ FDIL } & \multicolumn{2}{|c|}{ PFDIL } & \multicolumn{2}{|c|}{ DEBTL } & \multicolumn{2}{|c|}{ PDETL } \\
\hline & (1) & (2) & (3) & (4) & (5) & (6) & (7) & (8) \\
\hline & $\begin{array}{c}-0.048^{* * *} \\
(0.022)\end{array}$ & $\begin{array}{c}-0.047 * * \\
(0.022)\end{array}$ & $\begin{array}{c}-0.039^{*} \\
(0.022)\end{array}$ & $\begin{array}{l}-0.038^{*} \\
(0.022)\end{array}$ & $\begin{array}{c}-0.042 * \\
(0.022)\end{array}$ & $\begin{array}{c}-0.041 * \\
(0.022)\end{array}$ & $\begin{array}{c}-0.042^{*} \\
(0.022)\end{array}$ & $\begin{array}{l}-0.041^{*} \\
(0.022)\end{array}$ \\
\hline L.M2/REG & -0.049 & -0.048 & 0.039 & 0.039 & 0.039 & 0.038 & -0.023 & -0.023 \\
\hline & (0.209) & (0.209) & $(0.211)$ & $(0.211)$ & $(0.211)$ & $(0.211)$ & (0.209) & (0.209) \\
\hline L.CLAIMG & 0.000 & 0.000 & 0.000 & 0.000 & 0.000 & 0.000 & 0.000 & 0.000 \\
\hline & $(0.000)$ & $(0.000)$ & $(0.000)$ & $(0.000)$ & $(0.000)$ & $(0.000)$ & $(0.000)$ & $(0.000)$ \\
\hline L.LIFE & $\begin{array}{c}-0.063 * * * \\
(0.021)\end{array}$ & $\begin{array}{c}-0.063 * * * \\
(0.021)\end{array}$ & $\begin{array}{c}-0.065^{* * * *} \\
(0.024)\end{array}$ & $\begin{array}{c}-0.065^{* * * *} \\
(0.024)\end{array}$ & $\begin{array}{c}-0.072^{* * * *} \\
(0.022)\end{array}$ & $\begin{array}{c}-0.071 * * * * \\
(0.021)\end{array}$ & $\begin{array}{c}-0.064 * * * \\
(0.021)\end{array}$ & $\begin{array}{c}-0.064 * * * * \\
(0.021)\end{array}$ \\
\hline L.CPRIV & $\begin{array}{c}0.468 * * \\
(0.217)\end{array}$ & $\begin{array}{c}0.486^{* *} \\
(0.216)\end{array}$ & $\begin{array}{c}0.634 * * * \\
(0.240)\end{array}$ & $\begin{array}{c}0.640 * * * \\
(0.239)\end{array}$ & $\begin{array}{c}0.485^{* *} \\
(0.217)\end{array}$ & $\begin{array}{c}0.500 * * \\
(0.217)\end{array}$ & $\begin{array}{c}0.476^{* *} \\
(0.215)\end{array}$ & $\begin{array}{c}0.493 * * \\
(0.215)\end{array}$ \\
\hline L.LIA1 & $\begin{array}{l}\mathbf{- 0 . 2 6 9 *} \\
(0.143)\end{array}$ & $\begin{array}{l}-\mathbf{0 . 2 6 5 *} \\
(0.142)\end{array}$ & & & $\begin{array}{c}\mathbf{0 . 6 3 4} * * * * \\
(0.200)\end{array}$ & $\begin{array}{c}\mathbf{0 . 6 1 4} * * * \\
(0.200)\end{array}$ & & \\
\hline Z-SCOEXE & $\begin{array}{c}\mathbf{0 . 7 3 6}^{* * *} \\
(0.311)\end{array}$ & & $\begin{array}{l}\mathbf{0 . 6 2 9 *} \\
(0.327)\end{array}$ & & $\begin{array}{l}\text { 0.541* } \\
(0.325)\end{array}$ & & $\begin{array}{l}\text { 0.614* } \\
(0.314)\end{array}$ & \\
\hline Z-SCOEXA & & $\begin{array}{c}\mathbf{0 . 9 1 5 * * * *} \\
(0.341)\end{array}$ & & $\begin{array}{c}\mathbf{0 . 7 4 0} * * \\
(0.356)\end{array}$ & & $\begin{array}{l}\mathbf{0 . 6 7 2 *} \\
(0.352)\end{array}$ & & $\begin{array}{c}\mathbf{0 . 7 6 9} * * \\
(0.343)\end{array}$ \\
\hline L. LIA2 & & & $\begin{array}{c}\mathbf{- 0 . 7 9 4} * * * \\
(0.205)\end{array}$ & $\begin{array}{c}\mathbf{- 0 . 7 7 1} * * * * \\
(0.204)\end{array}$ & & & $\begin{array}{c}\mathbf{1 . 6 6 4} * * * * \\
(0.613)\end{array}$ & $\begin{array}{c}\mathbf{1 . 6 1 6}^{* * * *} \\
(0.611)\end{array}$ \\
\hline Constant & $\begin{array}{l}-0.376 \\
(1.227)\end{array}$ & $\begin{array}{l}-0.449 \\
(1.211)\end{array}$ & $\begin{array}{c}0.539 \\
(1.445)\end{array}$ & $\begin{array}{c}0.462 \\
(1.430)\end{array}$ & $\begin{array}{c}-3.190 * * \\
(1.511)\end{array}$ & $\begin{array}{c}-3.181 * * \\
(1.501)\end{array}$ & $\begin{array}{c}-8.197 * * * \\
(3.092)\end{array}$ & $\begin{array}{c}-8.053 * * * \\
(3.073)\end{array}$ \\
\hline Observations & 1,569 & 1,569 & 1,569 & 1,569 & 1,586 & 1,586 & 1,586 & 1,586 \\
\hline $\begin{array}{l}\text { Wald Test } \\
\text { Statistic }\end{array}$ & 33.13 & 34.89 & 39.71 & 40.79 & 37.77 & 39.09 & 35.20 & 36.79 \\
\hline Log-Likelihood & -321.3 & -320.6 & -313.2 & -312.9 & -317.5 & -317.1 & -318.7 & -318.2 \\
\hline $\begin{array}{l}\text { Likelihood Ratio } \\
\text { Test }\end{array}$ & 22.99 & 21.52 & 34.46 & 32.57 & 26.26 & 24.85 & 26.02 & 24.38 \\
\hline
\end{tabular}

Notes: Dependent variable is a banking crisis dummy. Marginal effects are reported. Symbols *, ** and $* * *$ indicate statistical significance at $10 \%, 5 \%$, and $1 \%$, respectively. Standard errors are presented below the corresponding coefficient. 
iv) Table $\mathrm{F}$ in appendix shows the results of the regressions which included four alternative control variables ${ }^{10}$, namely GDP per capita (GDP/C), government spending to GDP (GOV), the lack of price stability (INF) and political rights (POL: $1=$ most free and $7=$ least free) and excluded CLAIMG and LIFE. Once again, our basic results and conclusions remain unchanged after this last robustness test.

\section{Conclusion}

This study examined the determinants of the occurrence of banking crises in developing countries, focusing on the impact of the nature of external liabilities and exchange rate stability. For this propose, we used a logit panel model, including 67 developing countries observed between 1972 and 2011, and a set of alternative estimation methods (logit fixedeffects and probit random-effects), as well as several robustness tests. We found that FDI liabilities reduce banking crises occurrence, whereas debt liabilities increase them. In addition, the occurrence of banking crises decreases with the stability of the exchange rate. Economic prosperity and human capital quality are the robust decreasing factors of banking crises occurrence, but private credits boost the latter. The results led us to conclude that less volatile exchange rate regimes and capital control are the right political choice for developing countries to combat financial instability in the context of financial globalization. These recommendations are in line with those of Gaies et al. (2018) from their study of the triplet "financial globalization, instability and growth" in developing countries.

\section{Appendix}

\section{Summary statistics}

\begin{tabular}{|l|c|c|c|c|c|}
\hline Variable & Obs & Mean & Std. Dev. & Min & Max \\
\hline BCRISIS & 2680 & 0,57 & 0,23 & 0,00 & 1,00 \\
\hline FDIL & 2357 & 0,26 & 0,74 & 0,00 & 15,23 \\
\hline PFDIL & 2357 & 0,24 & 0,19 & 0,00 & 1,00 \\
\hline DEBTL & 2357 & 0,73 & 1,05 & 0,00 & 20,83 \\
\hline PDEBTL & 2357 & 0,75 & 0,19 & 0,00 & 1,00 \\
\hline CPRIVET & 1809 & 17,35 & 13,17 & 0,00 & 109,09 \\
\hline EXSE & 2387 & 0,25 & 0,32 & 0,00 & 1,00 \\
\hline EXSA & 2385 & 0,26 & 0,31 & 0,00 & 1,00 \\
\hline Z-SCOEXE & 2387 & 0,24 & 0,49 & 0,00 & 7,76 \\
\hline Z-SCOEXA & 2385 & 0,21 & 0,44 & 0,00 & 6,55 \\
\hline GROW & 2356 & 3,48 & 7,10 & $-64,05$ & 106,28 \\
\hline M2/REG & 2116 & $-0,02$ & 0,54 & $-7,02$ & 2,62 \\
\hline CLAIMG & 2174 & 0,22 & 2,70 & $-0,70$ & 110,46 \\
\hline LIFE & 2680 & 56,58 & 9,61 & 19,50 & 77,16 \\
\hline INF & 1911 & 4,73 & 0,28 & 4,41 & 10,08 \\
\hline GDP/C & 2391 & 6,51 & 0,78 & 3,91 & 8,34 \\
\hline GOV & 2170 & 15,14 & 7,75 & 1,38 & 69,54 \\
\hline POLI & 2514 & 5,60 & 2,10 & 1,00 & 7,00 \\
\hline
\end{tabular}

Data from 1972 to 2011 , including the 67 developing countries listed on the side.

\section{Sample countries}

Basic sample (67 countries)

Albania, Chad, Georgia, Kiribati, Niger, Sudan, Armenia, Comoros, Ghana, Lesotho, Nigeria, Swaziland, Bangladesh, Rep. Demo of Congo, Guatemala, Liberia,, Syria, Belize, Republic of Congo, Guinea, Madagascar, Papua New Guinea, Tajikistan, Benin, Côte d'Ivoire, Guinea-Bissau, Malawi, Paraguay, Tanzania, Bhutan, Djibouti, Guyana, Mali, Tonga, Bolivia, Haiti, Mauritania, Rwanda, Uganda, Burkina Faso, Salvador, Honduras, Moldova, Samoa, Uzbekistan, Burundi, Eritrea, Mongolia, Senegal, Vanuatu, Cambodia, Ethiopia, Mozambique, Sierra Leone, Vietnam, Cameroon, Fiji, Iraq, Nepal, Solomon Islands, Zambia Rep., Central African Republic, Gambia, Kenya, Nicaragua, Sri Lanka, Zimbabwe.

Classification by Region

East Asia and Pacific (10 countries)

Cambodia Fiji, Kiribati, Mongolia, Papua New Guinea, Samoa, Solomon Islands, Tonga, Vanuatu, Vietnam.

Europe and Central Asia (7 countries)

Albania, Armenia, Georgia, Moldova, Tajikistan, Uzbekistan, Belize.

South Asia (4 countries)

Bangladesh, Bhutan, Nepal, Sri Lanka.

Latin America and The Caribbean (8 countries)

Bolivia, El Salvador, Guatemala, Guyana, Haiti, Honduras, Nicaragua, Paraguay. Middle East and North Africa (3 countries)

Djibouti, Iraq, Syrian Arab Republic.

Sub-Saharan Africa (35 countries)

Benin, Burkina Faso, Burundi, Cameroon, Central African Rep., Chad, Comoros, Congo. Dem. Rep. of, Congo. Republic of, Côte d'Ivoire, Eritrea, Ethiopia, Gambia. The, Ghana, Guinea, Guinea Bissau, Kenya, Lesotho, Liberia, Madagascar, Malawi, Mali, Mauritania, Mozambique, Niger, Nigeria, Rwanda, Senegal, Sierra Leone, Sudan, Swaziland, Tanzania, Uganda, Zambia, Zimbabwe.

\footnotetext{
${ }^{10} \mathrm{GDP} / \mathrm{C}$ (in $\log$ ), GOV and INF (log (100+inflation rate)) are extracted from the WDI database and POL $(1=$ most free and $7=$ least free) is the Freedom House's index of political rights. These variables are used by recent literature, Joyce (2011) and Hamdi et al. (2014) among others.
} 
Table A Robustness estimations: excluding 1982-1986

\begin{tabular}{|c|c|c|c|c|c|c|c|c|}
\hline \multirow{3}{*}{$\begin{array}{l}\text { LIA1/ LIA2: } \\
\text { L.GROW }\end{array}$} & \multicolumn{2}{|c|}{ FDIL } & \multicolumn{2}{|c|}{ PFDIL } & \multicolumn{2}{|c|}{ DEBTL } & \multicolumn{2}{|c|}{ PDETL } \\
\hline & (1) & (2) & (3) & (4) & (5) & (6) & (7) & (8) \\
\hline & $\begin{array}{c}-0.057 * * * * \\
(0.021)\end{array}$ & $\begin{array}{c}-0.054 * * \\
(0.022)\end{array}$ & $\begin{array}{c}-0.048^{* *} \\
(0.022)\end{array}$ & $\begin{array}{c}-0.046^{* * *} \\
(0.022)\end{array}$ & $\begin{array}{c}-0.050^{* *} \\
(0.022)\end{array}$ & $\begin{array}{c}-0.049 * * \\
(0.022)\end{array}$ & $\begin{array}{c}-0.050^{* *} \\
(0.022)\end{array}$ & $\begin{array}{c}-0.048 * * \\
(0.022)\end{array}$ \\
\hline \multirow[t]{2}{*}{ L.M2/REG } & 0.000 & -0.007 & 0.072 & 0.062 & 0.058 & 0.047 & 0.019 & 0.015 \\
\hline & $(0.211)$ & $(0.211)$ & $(0.212)$ & $(0.212)$ & $(0.212)$ & $(0.212)$ & $(0.211)$ & $(0.211)$ \\
\hline \multirow[t]{2}{*}{ L.CLAIMG } & 0.001 & 0.001 & 0.001 & 0.001 & 0.001 & 0.001 & 0.001 & 0.001 \\
\hline & $(0.001)$ & $(0.001)$ & $(0.001)$ & $(0.001)$ & $(0.001)$ & $(0.001)$ & $(0.001)$ & $(0.001)$ \\
\hline \multirow[t]{2}{*}{ L.LIFE } & $-0.063 * * *$ & $-0.064 * * *$ & $-0.066 * * *$ & $-0.066^{* * * *}$ & $-0.068 * * *$ & $-0.067 * * *$ & $-0.063 * * *$ & $-0.064 * * *$ \\
\hline & $(0.021)$ & $(0.021)$ & $(0.024)$ & $(0.024)$ & $(0.022)$ & $(0.022)$ & $(0.021)$ & $(0.021)$ \\
\hline \multirow[t]{2}{*}{ L.CPRIV } & $0.397 *$ & $0.409 * *$ & $0.546^{* *}$ & $0.539 * *$ & $0.415^{* *}$ & $0.415^{* *}$ & $0.410^{* *}$ & $0.417 * *$ \\
\hline & $(0.208)$ & $(0.208)$ & $(0.228)$ & $(0.226)$ & $(0.208)$ & $(0.208)$ & $(0.206)$ & $(0.206)$ \\
\hline L.LIA1 & $\begin{array}{c}\mathbf{- 0 . 3 2 4} * * \\
(0.145)\end{array}$ & $\begin{array}{c}\mathbf{- 0 . 3 2 6}^{* * *} \\
(0.143)\end{array}$ & & & $\begin{array}{c}\mathbf{0 . 4 9 4} * * \\
(0.205)\end{array}$ & $\begin{array}{c}\text { 0.439** } \\
(0.206)\end{array}$ & & \\
\hline L.EXSE & $-2.164 * * *$ & & $-1.849 * *$ & & $-1.710^{* *}$ & & $-1.908 * *$ & \\
\hline \multirow[t]{2}{*}{ L. EXSA } & $(0.818)$ & $-2.670 * * *$ & $(0.806)$ & $-2.167 * *$ & $(0.824)$ & $-2.102 * *$ & $(0.797)$ & $-2.345 * * * *$ \\
\hline & & $(0.876)$ & & $(0.848)$ & & $(0.874)$ & & $(0.849)$ \\
\hline L. LIA2 & & & $\begin{array}{c}\mathbf{- 0 . 7 2 1} * * * * \\
(0.201)\end{array}$ & $\begin{array}{c}-\mathbf{- 0 . 6 6 9} * * * * \\
(0.195)\end{array}$ & & & $\begin{array}{l}\mathbf{1 . 4 7 4} * * \\
(0.593)\end{array}$ & $\begin{array}{l}1.396 * * \\
(0.584)\end{array}$ \\
\hline \multirow[t]{2}{*}{ Constant } & 0.550 & 0.648 & 1.185 & 1.190 & -2.151 & -1.874 & $-6.686^{* *}$ & $-6.259 * *$ \\
\hline & (1.196) & (1.193) & (1.356) & $(1.332)$ & (1.513) & $(1.512)$ & $(2.982)$ & $(2.936)$ \\
\hline Observations & 1,523 & 1,523 & 1,523 & 1,523 & 1,538 & 1,538 & 1,538 & 1,538 \\
\hline $\begin{array}{l}\text { Wald Test } \\
\text { Statistic }\end{array}$ & 34.84 & 36.89 & 38.81 & 40.56 & 36.08 & 37.38 & 36.15 & 37.95 \\
\hline Log-Likelihood & -314.2 & -312.4 & -308.7 & -307.9 & -313.9 & -312.9 & -313.3 & -311.9 \\
\hline $\begin{array}{l}\text { Likelihood } \\
\text { Ratio Test }\end{array}$ & 22.42 & 22.28 & 30.74 & 29.41 & 23.25 & 22.52 & 23.36 & 22.70 \\
\hline
\end{tabular}

Table B Robustness estimations: excluding 1997-2001

\begin{tabular}{|c|c|c|c|c|c|c|c|c|}
\hline \multirow[t]{2}{*}{ LIA1/ LIA2: } & \multicolumn{2}{|c|}{ FDIL } & \multicolumn{2}{|c|}{ PFDIL } & \multicolumn{2}{|c|}{ DEBTL } & \multicolumn{2}{|c|}{ PDETL } \\
\hline & (1) & (2) & (3) & (4) & (5) & (6) & (7) & (8) \\
\hline L.GROW & $\begin{array}{c}-0.053^{* * *} \\
(0.022)\end{array}$ & $\begin{array}{c}-0.049^{* *} \\
(0.022)\end{array}$ & $\begin{array}{l}-0.040^{*} \\
(0.023)\end{array}$ & $\begin{array}{l}-0.037 \\
(0.023)\end{array}$ & $\begin{array}{l}-0.038^{*} \\
(0.022)\end{array}$ & $\begin{array}{l}-0.037 \\
(0.023)\end{array}$ & $\begin{array}{l}-0.044^{*} \\
(0.022)\end{array}$ & $\begin{array}{l}-0.041^{*} \\
(0.023)\end{array}$ \\
\hline L.M2/REG & 0.013 & 0.012 & 0.095 & 0.084 & 0.080 & 0.072 & 0.032 & 0.030 \\
\hline & $(0.214)$ & $(0.214)$ & $(0.217)$ & $(0.216)$ & $(0.216)$ & $(0.215)$ & $(0.215)$ & $(0.215)$ \\
\hline L.CLAIMG & 0.001 & 0.001 & 0.001 & 0.001 & 0.001 & 0.001 & 0.001 & 0.001 \\
\hline & $(0.001)$ & (0.001) & $(0.001)$ & $(0.001)$ & $(0.001)$ & $(0.001)$ & $(0.001)$ & (0.001) \\
\hline L.LIFE & $-0.056^{* * *}$ & $-0.056^{* *}$ & $-0.060^{* *}$ & $-0.061 * *$ & $-0.066^{* * * *}$ & $-0.066 * * *$ & $-0.058^{* *}$ & $-0.058 * * *$ \\
\hline & $(0.023)$ & $(0.022)$ & $(0.026)$ & $(0.025)$ & $(0.024)$ & $(0.023)$ & $(0.023)$ & $(0.022)$ \\
\hline L.CPRIV & $\begin{array}{c}0.588 * * * \\
(0.228)\end{array}$ & $\begin{array}{c}0.588 * * * \\
(0.226)\end{array}$ & $\begin{array}{c}0.835^{* * * *} \\
(0.259)\end{array}$ & $\begin{array}{c}0.812 * * * \\
(0.256)\end{array}$ & $\begin{array}{c}0.687 * * * \\
(0.238)\end{array}$ & $\begin{array}{c}0.682 * * * \\
(0.238)\end{array}$ & $\begin{array}{c}0.621 * * * \\
(0.230)\end{array}$ & $\begin{array}{c}0.626^{* * * *} \\
(0.230)\end{array}$ \\
\hline L.LIA1 & $\begin{array}{l}-\mathbf{- 0 . 3 2 8} * * \\
(0.153)\end{array}$ & $\begin{array}{c}-\mathbf{- 0 . 3 2 5} * * \\
(0.150)\end{array}$ & & & $\begin{array}{c}\mathbf{0 . 7 8 3} * * * * \\
(0.233)\end{array}$ & $\begin{array}{c}\mathbf{0 . 7 2 3} * * * \\
(0.234)\end{array}$ & & \\
\hline L.EXSE & $\begin{array}{c}\mathbf{- 3 . 0 0 5} * * * \\
(1.013)\end{array}$ & & $\begin{array}{c}\mathbf{- 2 . 5 6 2} * * * \\
(0.954)\end{array}$ & & $\begin{array}{c}\mathbf{- 2 . 3 1 8} * * \\
(0.979)\end{array}$ & & $\begin{array}{c}\mathbf{- 2 . 6 0 1} * * * \\
(0.963)\end{array}$ & \\
\hline L. EXSA & & $-3.681 * * *$ & & $-2.960 * * *$ & & $-2.768 * * *$ & & $-3.187 * * *$ \\
\hline & & (1.092) & & $(1.019)$ & & (1.049) & & $(1.046)$ \\
\hline L. LIA2 & & & $\begin{array}{c}-\mathbf{- 0 . 8 9 9} * * * \\
(0.219)\end{array}$ & $\begin{array}{c}-\mathbf{- 0 . 8 2 5} * * * * \\
(0.211)\end{array}$ & & & $\begin{array}{c}\mathbf{1 . 7 5 6}^{* * * *} \\
(0.631)\end{array}$ & $\begin{array}{c}1.662 * * * \\
(0.625)\end{array}$ \\
\hline Constant & -0.347 & -0.221 & 0.469 & 0.497 & $-4.267 * *$ & $-3.956^{* *}$ & $-8.796 * * *$ & $-8.272 * * *$ \\
\hline & (1.285) & (1.270) & (1.495) & (1.456) & (1.717) & (1.708) & (3.201) & (3.160) \\
\hline Observations & 1,515 & 1,515 & 1,515 & 1,515 & 1,530 & 1,530 & 1,530 & 1,530 \\
\hline $\begin{array}{l}\text { Wald Test } \\
\text { Statistic }\end{array}$ & 33.14 & 35.58 & 40.89 & 42.73 & 38.35 & 39.31 & 36.07 & 37.69 \\
\hline Log-Likelihood & -302.7 & -300.5 & -293.8 & -292.9 & -298.4 & -297.4 & -300.3 & -298.6 \\
\hline $\begin{array}{l}\text { Likelihood Ratio } \\
\text { Test }\end{array}$ & 26.16 & 25.52 & 38.24 & 35.90 & 30.04 & 28.80 & 28.12 & 26.85 \\
\hline
\end{tabular}

Notes: Dependent variable is a banking crisis dummy. Marginal effects are reported. Symbols $*, * *$ and $* * *$ indicate statistical significance at $10 \%, 5 \%$, and $1 \%$, respectively. Standard errors are presented below the corresponding coefficient. 
Table C Robustness estimations: excluding 2007-2011

\begin{tabular}{|c|c|c|c|c|c|c|c|c|}
\hline \multirow[t]{2}{*}{ LIA1/ LIA2: } & \multicolumn{2}{|c|}{ FDIL } & \multicolumn{2}{|c|}{ PFDIL } & \multicolumn{2}{|c|}{ DEBTL } & \multicolumn{2}{|c|}{ PDETL } \\
\hline & (1) & (2) & (3) & (4) & (5) & (6) & (7) & (8) \\
\hline L.GROW & $\begin{array}{c}-0.065^{* * *} \\
(0.021)\end{array}$ & $\begin{array}{c}-0.063^{* * * *} \\
(0.022)\end{array}$ & $\begin{array}{c}-0.057^{* *} \\
(0.022)\end{array}$ & $\begin{array}{c}-0.056^{* *} \\
(0.022)\end{array}$ & $\begin{array}{c}-0.057 * * * \\
(0.022)\end{array}$ & $\begin{array}{c}-0.056 * * \\
(0.022)\end{array}$ & $\begin{array}{c}-0.056 * * \\
(0.022)\end{array}$ & $\begin{array}{c}-0.055 * * \\
(0.023)\end{array}$ \\
\hline L.M2/REG & $\begin{array}{l}-0.047 \\
(0.213)\end{array}$ & $\begin{array}{l}-0.042 \\
(0.213)\end{array}$ & $\begin{array}{c}0.033 \\
(0.215)\end{array}$ & $\begin{array}{c}0.029 \\
(0.215)\end{array}$ & $\begin{array}{c}0.035 \\
(0.216)\end{array}$ & $\begin{array}{c}0.032 \\
(0.216)\end{array}$ & $\begin{array}{l}-0.000 \\
(0.215)\end{array}$ & $\begin{array}{c}0.000 \\
(0.215)\end{array}$ \\
\hline L.CLAIMG & $\begin{array}{c}0.001 \\
(0.001)\end{array}$ & $\begin{array}{c}0.001 \\
(0.001)\end{array}$ & $\begin{array}{c}0.001 \\
(0.001)\end{array}$ & $\begin{array}{c}0.001 \\
(0.001)\end{array}$ & $\begin{array}{c}0.001 \\
(0.000)\end{array}$ & $\begin{array}{c}0.001 \\
(0.000)\end{array}$ & $\begin{array}{c}0.001 \\
(0.000)\end{array}$ & $\begin{array}{c}0.001 \\
(0.000)\end{array}$ \\
\hline L.LIFE & $\begin{array}{c}-0.055 * * * \\
(0.021)\end{array}$ & $\begin{array}{c}-0.055 * * * \\
(0.021)\end{array}$ & $\begin{array}{c}-0.065^{* *} \\
(0.026)\end{array}$ & $\begin{array}{c}-0.065 * * * \\
(0.025)\end{array}$ & $\begin{array}{c}-0.061 * * * \\
(0.022)\end{array}$ & $\begin{array}{c}-0.061 * * * \\
(0.022)\end{array}$ & $\begin{array}{c}-0.064 * * * \\
(0.024)\end{array}$ & $\begin{array}{c}-0.064 * * * * \\
(0.024)\end{array}$ \\
\hline L.CPRIV & $\begin{array}{c}0.153 \\
(0.198)\end{array}$ & $\begin{array}{c}0.153 \\
(0.197)\end{array}$ & $\begin{array}{c}0.292 \\
(0.229)\end{array}$ & $\begin{array}{c}0.275 \\
(0.225)\end{array}$ & $\begin{array}{c}0.202 \\
(0.201)\end{array}$ & $\begin{array}{c}0.197 \\
(0.200)\end{array}$ & $\begin{array}{c}0.242 \\
(0.214)\end{array}$ & $\begin{array}{c}0.238 \\
(0.212)\end{array}$ \\
\hline L.LIA1 & $\begin{array}{l}-\mathbf{- 0 . 2 7 0 *} \\
(0.155)\end{array}$ & $\begin{array}{l}-\mathbf{0 . 2 6 6 *} \\
(0.152)\end{array}$ & & & $\begin{array}{c}\mathbf{0 . 5 7 6}^{* * * *} \\
(0.215)\end{array}$ & $\begin{array}{r}\mathbf{0 . 5 5 5} \text { *** } \\
(0.216)\end{array}$ & & \\
\hline L.EXSE & $\begin{array}{c}-\mathbf{2 . 1 7 0} * * * \\
(0.803)\end{array}$ & & $\begin{array}{c}\mathbf{- 1 . 8 3 4 * * *} \\
(0.801)\end{array}$ & & $\begin{array}{l}\mathbf{- 1 . 5 3 9 *} \\
(0.804)\end{array}$ & & $\begin{array}{c}\mathbf{- 1 . 8 0 0 * * *} \\
(0.792)\end{array}$ & \\
\hline L. EXSA & & $\begin{array}{c}-\mathbf{2 . 3 8 1} * * * * \\
(0.846)\end{array}$ & & $\begin{array}{c}\mathbf{- 1 . 8 4 4} * * \\
(0.829)\end{array}$ & & $\begin{array}{l}-\mathbf{- 1 . 5 9 8 *}^{*} \\
(0.833)\end{array}$ & & $\begin{array}{c}\mathbf{- 1 . 9 0 9 * *} \\
(0.815)\end{array}$ \\
\hline L. LIA2 & & & $\begin{array}{c}-\mathbf{- 0 . 8 2 1} * * * * \\
(0.256)\end{array}$ & $\begin{array}{c}-\mathbf{- 0 . 7 6 4} * * * * \\
(0.244)\end{array}$ & & & $\begin{array}{c}\mathbf{3 . 8 2 8}^{* * * * *} \\
(1.175)\end{array}$ & $\begin{array}{c}\mathbf{3 . 7 3 9}^{* * * * *} \\
(1.162)\end{array}$ \\
\hline Constant & $\begin{array}{l}-0.544 \\
(1.288)\end{array}$ & $\begin{array}{l}-0.487 \\
(1.265)\end{array}$ & $\begin{array}{l}-1.729 \\
(1.552)\end{array}$ & $\begin{array}{l}-1.539 \\
(1.507)\end{array}$ & $\begin{array}{c}0.445 \\
(1.172)\end{array}$ & $\begin{array}{c}0.446 \\
(1.164)\end{array}$ & $\begin{array}{c}1.002 \\
(1.357)\end{array}$ & $\begin{array}{c}1.049 \\
(1.344)\end{array}$ \\
\hline Observations & 1,282 & 1,282 & 1,282 & 1,282 & 1,299 & 1,299 & 1,299 & 1,299 \\
\hline $\begin{array}{l}\text { Wald Test } \\
\text { Statistic }\end{array}$ & 30.55 & 31.06 & 32.61 & 33.64 & 35.08 & 35.51 & 34.02 & 34.53 \\
\hline $\begin{array}{l}\text { Log- } \\
\text { Likelihood }\end{array}$ & -286.7 & -286.5 & -280.5 & -280.8 & -284.5 & -284.6 & -280.2 & -280.1 \\
\hline $\begin{array}{l}\text { Likelihood } \\
\text { Ratio Test }\end{array}$ & 16.90 & 15.99 & 25.52 & 23.86 & 18.04 & 17.23 & 23.77 & 22.54 \\
\hline
\end{tabular}

Figure A Banking crisis in developing countries between 1972 and 2011

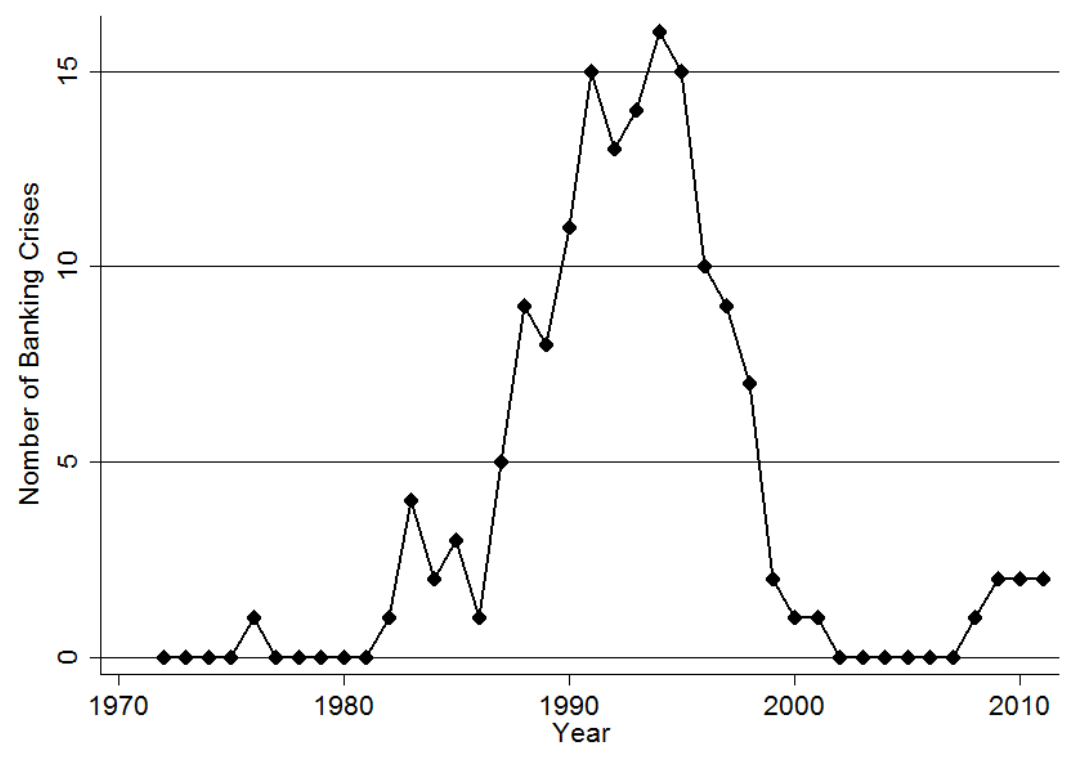

Notes: Calculation done by the authors on the basis of the banking crisis dummy variable by mean levels of the full sample (Systemic Banking Crises Database: An Update (IMF, 2012)). 
Table D Robustness estimations: omitting South Asia, East Asia and the Pacific

\begin{tabular}{|c|c|c|c|c|c|c|c|c|}
\hline \multirow{2}{*}{$\begin{array}{l}\text { LIA1/ LIA2: } \\
\text { L.GROW }\end{array}$} & \multicolumn{2}{|c|}{ FDIL } & \multicolumn{2}{|c|}{ PFDIL } & \multicolumn{2}{|c|}{ DEBTL } & \multicolumn{2}{|c|}{ PDETL } \\
\hline & $\begin{array}{c}(1) \\
-0.064 * * * \\
(0.021)\end{array}$ & $\begin{array}{c}(2) \\
-0.060 * * * \\
(0.022)\end{array}$ & $\begin{array}{c}(3) \\
-0.052 * * \\
(0.022)\end{array}$ & $\begin{array}{c}(4) \\
-0.050^{* *} \\
(0.022)\end{array}$ & $\begin{array}{c}(5) \\
-0.057 * * * \\
(0.022)\end{array}$ & $\begin{array}{c}(6) \\
-0.056^{* * * *} \\
(0.022)\end{array}$ & $\begin{array}{c}(7) \\
-0.054 * * \\
(0.022)\end{array}$ & $\begin{array}{c}(8) \\
-0.052 * * \\
(0.022)\end{array}$ \\
\hline L.M2/REG & $\begin{array}{c}0.001 \\
(0.214)\end{array}$ & $\begin{array}{c}0.002 \\
(0.215)\end{array}$ & $\begin{array}{c}0.091 \\
(0.216)\end{array}$ & $\begin{array}{c}0.086 \\
(0.216)\end{array}$ & $\begin{array}{c}0.081 \\
(0.216)\end{array}$ & $\begin{array}{c}0.076 \\
(0.216)\end{array}$ & $\begin{array}{c}0.041 \\
(0.214)\end{array}$ & $\begin{array}{c}0.043 \\
(0.215)\end{array}$ \\
\hline L.CLAIMG & $\begin{array}{c}0.001 \\
(0.001)\end{array}$ & $\begin{array}{c}0.001 \\
(0.001)\end{array}$ & $\begin{array}{c}0.001 \\
(0.001)\end{array}$ & $\begin{array}{c}0.001 \\
(0.001)\end{array}$ & $\begin{array}{c}0.001 \\
(0.001)\end{array}$ & $\begin{array}{c}0.001 \\
(0.001)\end{array}$ & $\begin{array}{c}0.001 \\
(0.001)\end{array}$ & $\begin{array}{c}0.001 \\
(0.001)\end{array}$ \\
\hline L.LIFE & $\begin{array}{c}-0.055^{* *} \\
(0.023)\end{array}$ & $\begin{array}{c}-0.055^{* *} \\
(0.023)\end{array}$ & $\begin{array}{c}-0.064 * * \\
(0.027)\end{array}$ & $\begin{array}{c}-0.064 * * \\
(0.027)\end{array}$ & $\begin{array}{c}-0.065^{* * *} \\
(0.023)\end{array}$ & $\begin{array}{c}-0.063^{* * *} \\
(0.022)\end{array}$ & $\begin{array}{c}-0.061 * * * \\
(0.023)\end{array}$ & $\begin{array}{c}-0.060^{* * *} \\
(0.023)\end{array}$ \\
\hline L.CPRIV & $\begin{array}{c}0.241 \\
(0.208)\end{array}$ & $\begin{array}{c}0.255 \\
(0.209)\end{array}$ & $\begin{array}{l}0.394 * \\
(0.236)\end{array}$ & $\begin{array}{l}0.396^{*} \\
(0.235)\end{array}$ & $\begin{array}{c}0.299 \\
(0.207)\end{array}$ & $\begin{array}{c}0.301 \\
(0.207)\end{array}$ & $\begin{array}{c}0.257 \\
(0.210)\end{array}$ & $\begin{array}{c}0.271 \\
(0.210)\end{array}$ \\
\hline L.LIA1 & $\begin{array}{c}-\mathbf{0 . 4 3 2} * * \\
(0.172)\end{array}$ & $\begin{array}{c}-\mathbf{0 . 4 4 5} * * * * \\
(0.172)\end{array}$ & & & $\begin{array}{l}\mathbf{0 . 4 9 2} * * \\
(0.203)\end{array}$ & $\begin{array}{l}\mathbf{0 . 4 3 9} \text { ** } \\
(0.203)\end{array}$ & & \\
\hline L.EXSE & $\begin{array}{c}\mathbf{- 2 . 1 6 1} * * * \\
(0.795)\end{array}$ & & $\begin{array}{c}-\mathbf{1 . 6 4 5} * * \\
(0.794)\end{array}$ & & $\begin{array}{l}-1.538 * \\
(0.807)\end{array}$ & & $\begin{array}{l}\mathbf{- 1 . 6 9 1} \text { ** } \\
(0.770)\end{array}$ & \\
\hline L. EXSA & & $\begin{array}{c}\mathbf{- 2 . 8 0 0} * * * \\
(0.893)\end{array}$ & & $\begin{array}{c}-\mathbf{2 . 0 7 0} * * \\
(0.856)\end{array}$ & & $\begin{array}{c}-\mathbf{2 . 0 4 5}^{* * *} \\
(0.884)\end{array}$ & & $\begin{array}{c}\mathbf{- 2 . 1 9 8} * * * \\
(0.842)\end{array}$ \\
\hline L. LIA2 & & & $\begin{array}{c}-\mathbf{- 0 . 9 4 0} * * * \\
(0.254)\end{array}$ & $\begin{array}{c}\mathbf{- 0 . 8 8 2} * * * * \\
(0.246)\end{array}$ & & & $\begin{array}{c}\mathbf{2 . 3 3 4} * * * * \\
(0.782)\end{array}$ & $\begin{array}{c}\mathbf{2 . 2 4 0} * * * * \\
(0.772)\end{array}$ \\
\hline Constant & $\begin{array}{c}0.899 \\
(1.295)\end{array}$ & $\begin{array}{l}0.982 \\
(1.306)\end{array}$ & $\begin{array}{c}2.048 \\
(1.585)\end{array}$ & $\begin{array}{c}1.978 \\
(1.559)\end{array}$ & $\begin{array}{l}-1.885 \\
(1.519)\end{array}$ & $\begin{array}{l}-1.673 \\
(1.514)\end{array}$ & $\begin{array}{c}-10.129 * * * \\
(3.726)\end{array}$ & $\begin{array}{c}-9.680 * * * \\
(3.673)\end{array}$ \\
\hline Observations & 1,238 & 1,238 & 1,238 & 1,238 & 1,249 & 1,249 & 1,249 & 1,249 \\
\hline $\begin{array}{l}\text { Wald Test } \\
\text { Statistic }\end{array}$ & 34.53 & 36.02 & 36.18 & 37.89 & 35.79 & 36.83 & 35.59 & 37.15 \\
\hline Log-Likelihood & -277.3 & -275.1 & -270.5 & -269.5 & -277.9 & -276.6 & -274.7 & -273.1 \\
\hline $\begin{array}{l}\text { Likelihood } \\
\text { Ratio Test }\end{array}$ & 14.96 & 15.59 & 24.88 & 24.05 & 14.12 & 13.76 & 18.12 & 17.74 \\
\hline
\end{tabular}

Table E Robustness estimations: omitting Latin America, Caribbean, Europe and Central Asia

\begin{tabular}{|c|c|c|c|c|c|c|c|c|}
\hline \multirow[t]{2}{*}{ LIA1/ LIA2: } & \multicolumn{2}{|c|}{ FDIL } & \multicolumn{2}{|c|}{ PFDIL } & \multicolumn{2}{|c|}{ DEBTL } & \multicolumn{2}{|c|}{ PDETL } \\
\hline & (1) & (2) & (3) & (4) & (5) & (6) & (7) & (8) \\
\hline L.GROW & $\begin{array}{c}-0.059 * * * \\
(0.022)\end{array}$ & $\begin{array}{c}-0.055^{* *} \\
(0.022)\end{array}$ & $\begin{array}{c}-0.049^{* *} \\
(0.023)\end{array}$ & $\begin{array}{c}-0.047^{* *} \\
(0.023)\end{array}$ & $\begin{array}{c}-0.052^{* *} \\
(0.022)\end{array}$ & $\begin{array}{c}-0.050^{* * *} \\
(0.022)\end{array}$ & $\begin{array}{c}-0.053^{* *} \\
(0.022)\end{array}$ & $\begin{array}{c}-0.050^{* * *} \\
(0.022)\end{array}$ \\
\hline L.M2/REG & -0.009 & -0.008 & 0.061 & 0.055 & 0.047 & 0.038 & 0.004 & 0.005 \\
\hline & $(0.216)$ & $(0.215)$ & $(0.218)$ & $(0.217)$ & $(0.217)$ & $(0.216)$ & $(0.216)$ & $(0.215)$ \\
\hline L.CLAIMG & -0.001 & -0.001 & -0.001 & -0.001 & -0.001 & -0.001 & -0.001 & -0.001 \\
\hline & $(0.003)$ & $(0.003)$ & $(0.003)$ & $(0.003)$ & $(0.003)$ & $(0.003)$ & $(0.003)$ & $(0.003)$ \\
\hline L.LIFE & $-0.047 *$ & $-0.047 *$ & $-0.056^{*}$ & $-0.056^{* *}$ & $-0.054 * *$ & $-0.052 * *$ & $-0.047 *$ & $-0.046^{*}$ \\
\hline & $(0.025)$ & $(0.025)$ & $(0.029)$ & $(0.028)$ & $(0.025)$ & $(0.025)$ & $(0.025)$ & $(0.025)$ \\
\hline L.CPRIV & $0.472^{* *}$ & $0.464 * *$ & $0.668 * * *$ & $0.644 * * *$ & $0.521^{* *}$ & $0.508^{* *}$ & $0.479 * *$ & $0.476^{* *}$ \\
\hline & $(0.226)$ & $(0.222)$ & $(0.251)$ & $(0.248)$ & $(0.229)$ & $(0.227)$ & $(0.223)$ & $(0.222)$ \\
\hline L.LIA1 & $\begin{array}{c}\mathbf{- 0 . 3 2 5 * *} \\
(0.154)\end{array}$ & $\begin{array}{c}-\mathbf{- 0 . 3 2 8} * * \\
(0.153)\end{array}$ & & & $\begin{array}{c}\mathbf{0 . 5 6 7 * * * *} \\
(0.217)\end{array}$ & $\begin{array}{r}\mathbf{0 . 4 8 7 * *} \\
(0.218)\end{array}$ & & \\
\hline L.EXSE & $-4.068 * * *$ & & $-3.240 * *$ & & $-3.161 * *$ & & $-3.505 * *$ & \\
\hline L. EXSA & $(1.472)$ & $-4.928 * * *$ & (1.351) & $-3.836 * * *$ & $(1.422)$ & $-3.937 * * *$ & (1.396) & $-4.341^{* * * *}$ \\
\hline & & $(1.449)$ & & $(1.376)$ & & (1.483) & & $(1.431)$ \\
\hline L. LIA2 & & & $\begin{array}{c}\mathbf{- 0 . 8 4 2} * * * * \\
(0.231)\end{array}$ & $\begin{array}{c}\mathbf{- 0 . 7 7 1} * * * * \\
(0.225)\end{array}$ & & & $\begin{array}{l}\mathbf{1 . 4 6 7 * * *} \\
(0.612)\end{array}$ & $\begin{array}{c}\mathbf{1 . 3 5 4} * * \\
(0.601)\end{array}$ \\
\hline Constant & -0.323 & -0.145 & 0.714 & 0.760 & $-3.318^{* *}$ & $-2.915^{*}$ & $-7.522 * *$ & $-6.903 * *$ \\
\hline & (1.366) & (1.347) & (1.616) & $(1.570)$ & $(1.680)$ & (1.664) & (3.121) & (3.059) \\
\hline Observations & 1,248 & 1,248 & 1,248 & 1,248 & 1,265 & 1,265 & 1,265 & 1,265 \\
\hline $\begin{array}{l}\text { Wald Test } \\
\text { Statistic }\end{array}$ & 28.68 & 32.41 & 35.07 & 37.31 & 31.28 & 32.77 & 30.14 & 32.71 \\
\hline Log-Likelihood & -284.8 & -282.2 & -278 & -276.7 & -283.4 & -282 & -283.8 & -281.7 \\
\hline $\begin{array}{l}\text { Likelihood Ratio } \\
\text { Test }\end{array}$ & 22.10 & 21.29 & 32.52 & 29.90 & 23.74 & 21.97 & 22.98 & 21.39 \\
\hline
\end{tabular}


Table F Robustness estimations: changing control variables

\begin{tabular}{|c|c|c|c|c|c|c|c|c|}
\hline \multirow{3}{*}{$\begin{array}{l}\text { LIA1/ LIA2: } \\
\text { L.GROW }\end{array}$} & \multicolumn{2}{|c|}{ FDIL } & \multicolumn{2}{|c|}{ PFDIL } & \multicolumn{2}{|c|}{ DEBTL } & \multicolumn{2}{|c|}{ PDETL } \\
\hline & (1) & (2) & (3) & (4) & (5) & (6) & (7) & (8) \\
\hline & $\begin{array}{c}-0.072 * * * \\
(0.024)\end{array}$ & $\begin{array}{c}-0.069 * * * \\
(0.024)\end{array}$ & $\begin{array}{c}-0.063 * * * \\
(0.024)\end{array}$ & $\begin{array}{c}-0.062 * * \\
(0.024)\end{array}$ & $\begin{array}{c}-0.067 * * * \\
(0.024)\end{array}$ & $\begin{array}{c}-0.066 * * * \\
(0.024)\end{array}$ & $\begin{array}{c}-0.067 * * * * \\
(0.024)\end{array}$ & $\begin{array}{c}-0.064 * * * \\
(0.024)\end{array}$ \\
\hline L.GDP/C & $\begin{array}{c}0.321 \\
(0.339)\end{array}$ & $\begin{array}{c}0.356 \\
(0.341)\end{array}$ & $\begin{array}{c}0.579 \\
(0.402)\end{array}$ & $\begin{array}{c}0.588 \\
(0.399)\end{array}$ & $\begin{array}{c}0.190 \\
(0.327)\end{array}$ & $\begin{array}{c}0.214 \\
(0.327)\end{array}$ & $\begin{array}{c}0.376 \\
(0.341)\end{array}$ & $\begin{array}{c}0.402 \\
(0.341)\end{array}$ \\
\hline L.M2/REG & $\begin{array}{c}0.106 \\
(0.246)\end{array}$ & $\begin{array}{c}0.103 \\
(0.245)\end{array}$ & $\begin{array}{c}0.196 \\
(0.248)\end{array}$ & $\begin{array}{c}0.186 \\
(0.247)\end{array}$ & $\begin{array}{c}0.179 \\
(0.248)\end{array}$ & $\begin{array}{c}0.171 \\
(0.247)\end{array}$ & $\begin{array}{c}0.140 \\
(0.246)\end{array}$ & $\begin{array}{c}0.137 \\
(0.246)\end{array}$ \\
\hline L.CPRIV & $\begin{array}{l}0.535 \text { ** } \\
(0.252)\end{array}$ & $\begin{array}{l}0.529 * * \\
(0.251)\end{array}$ & $\begin{array}{l}0.682 * * \\
(0.274)\end{array}$ & $\begin{array}{l}0.665^{* *} \\
(0.272)\end{array}$ & $\begin{array}{l}0.562 * * \\
(0.253)\end{array}$ & $\begin{array}{r}0.554^{* *} \\
(0.253)\end{array}$ & $\begin{array}{l}0.549 * * \\
(0.251)\end{array}$ & $\begin{array}{l}0.545^{* *} \\
(0.251)\end{array}$ \\
\hline L.GOV & $\begin{array}{l}-0.209 \\
(0.433)\end{array}$ & $\begin{array}{l}-0.240 \\
(0.435)\end{array}$ & $\begin{array}{l}-0.386 \\
(0.466)\end{array}$ & $\begin{array}{l}-0.404 \\
(0.467)\end{array}$ & $\begin{array}{l}-0.244 \\
(0.427)\end{array}$ & $\begin{array}{l}-0.272 \\
(0.428)\end{array}$ & $\begin{array}{l}-0.296 \\
(0.433)\end{array}$ & $\begin{array}{l}-0.318 \\
(0.434)\end{array}$ \\
\hline L.INF & $\begin{array}{c}0.003 \\
(0.002)\end{array}$ & $\begin{array}{c}0.003 \\
(0.002)\end{array}$ & $\begin{array}{c}0.003 \\
(0.002)\end{array}$ & $\begin{array}{c}0.003 \\
(0.002)\end{array}$ & $\begin{array}{l}0.003 * \\
(0.002)\end{array}$ & $\begin{array}{l}0.003 * \\
(0.002)\end{array}$ & $\begin{array}{c}0.003 \\
(0.002)\end{array}$ & $\begin{array}{c}0.003 \\
(0.002)\end{array}$ \\
\hline L.POL & $\begin{array}{l}0.222 * * \\
(0.092)\end{array}$ & $\begin{array}{l}0.202 * * \\
(0.092)\end{array}$ & $\begin{array}{c}0.227 * * \\
(0.096)\end{array}$ & $\begin{array}{c}0.211^{* *} \\
(0.096)\end{array}$ & $\begin{array}{c}0.248 * * * \\
(0.093)\end{array}$ & $\begin{array}{l}0.235 * * \\
(0.093)\end{array}$ & $\begin{array}{c}0.241 * * * * \\
(0.092)\end{array}$ & $\begin{array}{l}0.227 * * \\
(0.092)\end{array}$ \\
\hline L.LIA1 & $\begin{array}{c}-\mathbf{0 . 4 1 9 * *} \\
(0.174)\end{array}$ & $\begin{array}{c}-\mathbf{- 0 . 4 1 9 * * *} \\
(0.173)\end{array}$ & & & $\begin{array}{c}\mathbf{0 . 4 7 1} * * \\
(0.223)\end{array}$ & $\begin{array}{c}\mathbf{0 . 4 4 5 * *} \\
(0.222)\end{array}$ & & \\
\hline L.EXSE & $\begin{array}{c}-\mathbf{- 2 . 1 6 4 * * * *} \\
(0.796)\end{array}$ & & $\begin{array}{c}\mathbf{- 1 . 8 3 9 * * *} \\
(0.807)\end{array}$ & & $\begin{array}{c}\mathbf{- 1 . 7 8 0 * * *} \\
(0.817)\end{array}$ & & $\begin{array}{c}\mathbf{- 1 . 9 1 8 * *}^{*} \text { (0.787) }\end{array}$ & \\
\hline L. EXSA & & $\begin{array}{c}\mathbf{- 2 . 4 9 6 * * * *} \\
(0.850)\end{array}$ & & $\begin{array}{c}\mathbf{- 2 . 0 2 5 * *} \\
(0.846)\end{array}$ & & $\begin{array}{c}\mathbf{- 2 . 0 5 9 * * *} \\
(0.867)\end{array}$ & & $\begin{array}{c}\mathbf{- 2 . 2 2 9} * * * * \\
(0.837)\end{array}$ \\
\hline L.LIA12 & & & $\begin{array}{c}-\mathbf{- 0 . 8 6 0} \text { ***** } \\
(0.243)\end{array}$ & $\begin{array}{c}\mathbf{- 0 . 8 1 9 * * * *} \\
(0.238)\end{array}$ & & & $\begin{array}{l}\mathbf{1 . 6 3 7 * *} \\
(0.682)\end{array}$ & $\begin{array}{c}\mathbf{1 . 6 1 4} * * \\
(0.676)\end{array}$ \\
\hline Constant & $\begin{array}{c}-6.191 * * * \\
(2.378)\end{array}$ & $\begin{array}{c}-6.153 * * * \\
(2.380)\end{array}$ & $\begin{array}{c}-6.849 * * \\
(2.711)\end{array}$ & $\begin{array}{c}-6.788 * * \\
(2.689)\end{array}$ & $\begin{array}{c}-8.490 \text { **** } \\
(2.718)\end{array}$ & $\begin{array}{c}-8.320 * * * \\
(2.713)\end{array}$ & $\begin{array}{c}-14.565 * * * \\
(4.371)\end{array}$ & $\begin{array}{c}-14.433 * * * \\
(4.356)\end{array}$ \\
\hline Observations & 1,436 & 1,436 & 1,436 & 1,436 & 1,453 & 1,453 & 1,453 & 1,453 \\
\hline $\begin{array}{l}\text { Wald Test } \\
\text { Statistic }\end{array}$ & 33.63 & 34.39 & 37.35 & 37.94 & 32.85 & 33.12 & 33.62 & 34.22 \\
\hline $\begin{array}{l}\text { Log- } \\
\text { Likelihood }\end{array}$ & -272.3 & -271.5 & -267.1 & -266.8 & -273.2 & -272.6 & -272 & -271.2 \\
\hline $\begin{array}{l}\text { Likelihood } \\
\text { Ratio Test }\end{array}$ & 28.54 & 28.99 & 37.08 & 36.82 & 27.67 & 27.80 & 28.17 & 28.32 \\
\hline
\end{tabular}




\section{References}

Ahrend, R., \& Goujard, A. (2014). Drivers of systemic banking crises: The role of financial account structure and financial integration. International Finance, 17(3), 135-160.

Aizenman, J., Chinn, M.D., \& Ito, H. (2008). Assessing the emerging global financial architecture: Measuring the trilemma's configurations over time. NBER Working Papers, 14533.

Aizenman, J., Chinn, M.D., \& Ito, H. (2016). Monetary policy spillovers and the trilemma in the new normal: Periphery country sensitivity to core country conditions. Journal of International Money and Finance, 68, 298-330.

Babecký, J., Havránek, T., Matějů, J., Rusnák, M., Šmídková, K., \& Vašíček, B. (2014). Banking, debt, and currency crises in developed countries: Stylized facts and early warning indicators. Journal of Financial Stability, 15(C), 1-17.

Barro, R.J. (2001). Economic Growth in East Asia before and after the Financial Crises. NBER Working Papers, 8330.

Bekaert, G., \& Harvey, C.R. (2000). Foreign speculators and emerging equity markets. The Journal of Finance, 55(2), 565-613.

Bordo, M.D. (2018). Reflections on the Evolution of Financial Crises: Theory, History and Empirics. In Coping with Financial Crises (pp. 1-15). Springer, Singapore.

Broner, F., Martin, A., \& Ventura, J. (2010). Sovereign risk and secondary markets. The American Economic Review, 100(4), 1523-1555.

Claessens, S., \& Kose, M.A. (2013). Financial Crises Explanations, Types, and Implications. International Monetary Fund, 13(28).

Davis, J.S., Mack, A., Phoa, W., \& Vandenabeele, A. (2016). Credit booms, banking crises, and the current account. Journal of International Money and Finance, 60(C), 360-377.

Frankel, J.A., \& Rose, A. (2002). An Estimate of the Effect of Common Currencies on Trade and Income. The Quarterly Journal of Economics, 117(2), 437-466.

Gaies, B., Goutte, S., \& Guesmi, K. What Interactions between Financial Globalization and Instability? - Growth in Developing Countries. Journal of International Development.

Gourinchas, P. and Obstfeld, M. (2012). Stories of the twentieth century for the twenty-first. American Economic Journal: Macroeconomics, 4(1), 226-65.

Hamdi, H., \& Jlassi, N.B. (2014). Financial liberalization, disaggregated capital flows and banking crisis: Evidence from developing countries. Economic Modelling, 41(C), 124-132.

Hsing, Y. (2012). Impacts of the trilemma policies on inflation, growth and volatility in Greece. International Journal of Economics and Financial Issues, 2(3), 373.

Joyce, J.P. (2011). Financial globalization and banking crises in emerging markets. Open Economies Review, 22(5), 875-895.

Lane, P.R., \& McQuade, P. (2014). Domestic credit growth and international capital flows. The Scandinavian Journal of Economics, 116(1), 218-252.

Lee, C.C., Lin, C. W., \& Zeng, J. H. (2016). Financial liberalization, insurance market, and the likelihood of financial crises. Journal of International Money and Finance, 62(C), 25-51.

McKinnon, R.I., \& Pill, H. (1996). Credible Liberalizations and International Capital Flows: The "Overborrowing Syndrome". In Financial Deregulation and Integration in East Asia, NBEREASE Volume 5 (pp. 7-50). University of Chicago Press, Chicago.

McKinnon, R.I., \& Pill, H. (1998). International overborrowing: a decomposition of credit and currency risks. World Development, 26(7), 1267-1282.

Reinhart, C.M., \& Rogoff, K.S. (2013). Banking crises: an equal opportunity menace. Journal of Banking \& Finance, 37(11), 4557-4573.

Rose, A.K. (2000). One money, one market: the effect of common currencies on trade. Economic Policy, 15(30), 08-45.

Wei, S.J. (2018). Managing Financial Globalization: Insights from the Recent Literature. NBER Working Papers, 24330. 